\title{
Téoros
}

Revue de recherche en tourisme

\section{Le tourisme des jeunes}

\section{Jean Stafford, Bruno Sarrasin et Haja Ramahatra}

Volume 20, numéro 2, été 2001

URI : https://id.erudit.org/iderudit/1071645ar

DOI : https://doi.org/10.7202/1071645ar

Aller au sommaire du numéro

Éditeur(s)

Université du Québec à Montréal

ISSN

0712-8657 (imprimé)

1923-2705 (numérique)

Découvrir la revue

Citer cette note

Stafford, J., Sarrasin, B. \& Ramahatra, H. (2001). Le tourisme des jeunes. Téoros, 20(2), 60-62. https://doi.org/10.7202/1071645ar d'utilisation que vous pouvez consulter en ligne.

https://apropos.erudit.org/fr/usagers/politique-dutilisation/ 


\section{Conjoncture}

\section{Le tourisme des jeunes}

\section{Jean Stafford, Bruno Sarrasin et Haja Ramahatra}

Cette chronique porte habituellement sur la conjoncture touristique; exceptionnellement, dans le présent numéro, nous allons traiter de sondages récents portant sur les comportements touristiques des jeunes américains et des jeunes canadiens.

es comportements touristiques des jeunes sont assez mécon

nus. Cette analyse n'est qu'une partie d'un rapport de recherche effectué sous la coordination du Bureau international du tourisme social-section des Amériques (BITS-Amériques) pour le compte de la Commission canadienne du tourisme, avec la collaboration scientifique du Centre international de formation et de recherche en tourisme (CIFORT) de l'École des sciences de la gestion de 1'Université du Québec à Montréal.

\section{La population étudiée}

L'enquête sur les activités et les préférences des jeunes en matière de voyages a été réalisée pour le compte de la Commission canadienne du tourisme et de différents ministères fédéraux et provinciaux. La collecte des données (les entrevues) s'est faite du 27 septembre 1999 au 16 avril 2000. L'enquête comportait un sondage téléphonique et un sondage postal effectués aux ÉtatsUnis et au Canada.

Le sondage téléphonique s'est fait auprès de 28397 répondants aux États-Unis et de 18385 au Canada ; il s'agissait de deux sondages stratifiés et aléatoires. Quant au sondage postal, le nombre des répondants était de 6405 aux États-Unis et de 5405 au Canada. La firme Price Waterhouse Coopers était responsable de la collecte des données aux États-Unis, tandis que Statistique Canada avait le mandat de la collecte des données au Canada. La marge d'erreur théorique sur les réponses à $95 \%$ (19 fois sur 20) est d'environ $2 \%$.

Pour les fins de cette étude sur les comportements touristiques des jeunes des États-Unis et du Canada, nous avons extrait le groupe des dix-huit - vingt-quatre ans de 1'échantillon original.

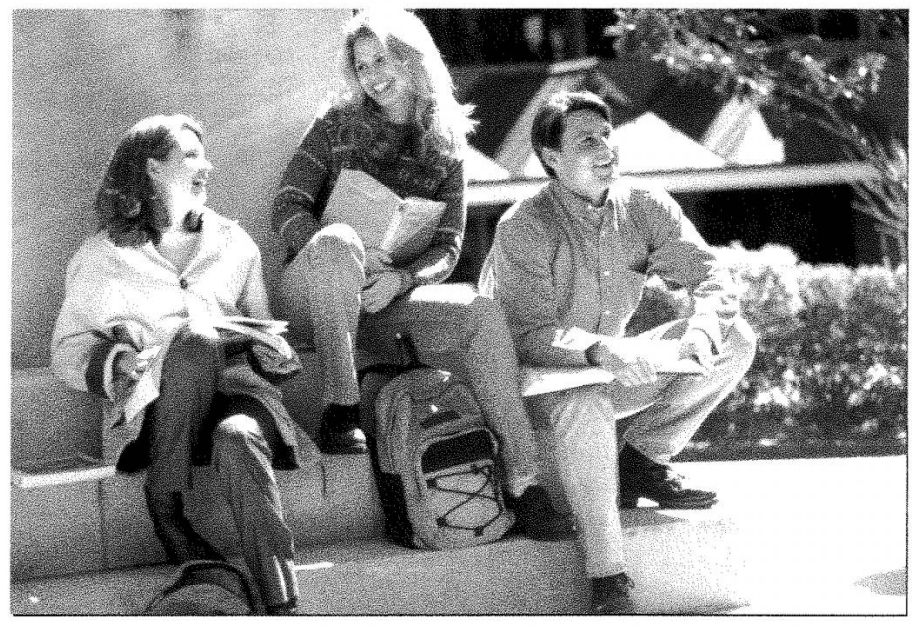

Le traitement et l'interprétation des données porteront uniquement sur ce groupe d'âge ; ce découpage correspond à celui effectué par différentes études internationales : il permet de comparer les comportements touristiques des jeunes de différents pays.

\section{Les vacances, les départs et la durée des voyages}

Au tableau 1 nous présentons le nombre de semaines de vacances auxquelles les répondants ont eu droit en 1999. Nous remarquons que les jeunes américains ont eu droit à moins de semaines de vacances que les jeunes canadiens : $33 \%$ des jeunes canadiens ont eu droit à trois semaines de vacances ou plus comparativement à seulement $19 \%$ des jeunes américains.

Au tableau 2, départs en voyages des jeunes selon le type de départ, nous constatons que les pourcentages des départs en voyages sont sensiblement les mêmes pour les jeunes américains et les jeunes canadiens. La très grande majorité des jeunes des deux pays ont un pourcentage de départ extrêmement élevé si l'on compare ces pourcentages à ceux des autres groupes d'âge.

Au tableau 3, nombre de jours passés en voyages d'agrément en 1999 , nous voyons que les jeunes canadiens voyagent plus longtemps que les jeunes américains, mais que les différences en termes de jours ne sont pas très importantes. 


\section{Les destinations de voyages ef la saisonnalité}

Le tableau 4 présente les principales destinations de voyages des jeunes américains et des jeunes canadiens dans les deux dernières années. Nous y voyons que les jeunes américains sont $12 \%$ à visiter le Canada alors que $31 \%$ des jeunes canadiens visitent les États-Unis ; nous pouvons ainsi constater que l'échange touristique est très inégal : l'attrait des États-Unis est plus élevé chez les Canadiens que l'attrait du Canada pour les Américains !

Les résultats du sondage ont démontré que les jeunes américains voyagent plus à l'étranger que les jeunes canadiens, mais qu'ils voyagent surtout dans des pays limitrophes: les principales destinations étrangères (à l'exception du Canada) des jeunes américains sont surtout le Mexique et les îles des Caraïbes. Les destinations étrangères (à l'exception des États-Unis) des jeunes canadiens sont très vastes : elles se divisent en trois parties presque égales (le tiers) entre les destinations Mexique-Caraïbes, l'Europe et les autres pays du monde.

Quant aux voyages réalisés au Canada par les jeunes des deux pays, le tableau 5 démontre que seules les trois principales provinces, celles qui ont le plus grand nombre des touristes, ont été considérées. Nous constatons ainsi que $44 \%$ des jeunes américains ont séjourné en Ontario, $25 \%$ en Colombie-Britannique et $17 \%$ au Québec. Les jeunes canadiens ont davantage tendance à voyager dans leur province respective, ce qui explique en partie les pourcentages inférieurs.

Par ailleurs, dans les intentions de voyages au cours des deux prochaines années, le Québec, comme destination touristique, arrive au deuxième rang chez les jeunes américains ( $52 \%$ de « Oui ») et seulement au quatrième rang chez les jeunes canadiens $(33 \%$ de « Oui »).

Au tableau 6 nous présentons la saisonnalité des départs en voyages dans les deux dernières années. Nous pouvons constater que les jeunes américains voyagent plus l'hiver et l'été et que les pourcentages des départs sont assez faibles au printemps et en automne. Chez les jeunes canadiens, l'été et l'hiver sont aussi les principales saisons, mais les pourcentages sont plus faibles que chez les jeunes américains. Les jeunes canadiens voyagent plus que les jeunes américains dans les saisons creuses : $19 \%$ au printemps et en automne.

\section{Les motifs de voyages et les sources d'information}

Au tableau 7 nous présentons les principaux motifs de voyages au Canada dans les deux dernières années. Nous pouvons constater que la très grande majorité des répondants américains et canadiens (plus de $90 \%$ ) voyagent au Canada pour l'agrément ou pour rencontrer des parents et des amis. Les jeunes canadiens voyagent plus pour affaires au Canada (15\%) que les jeunes américains $(8 \%)$. Les voyages effectués pour des raisons personnelles sont légèrement plus élevés chez les jeunes canadiens (17\%) que chez les jeunes américains (12\%). En ce qui con-

\begin{tabular}{|lcc|}
\hline & Winful & \\
\hline Nombre de semaines & $\begin{array}{c}\text { Américains } \\
\%\end{array}$ & $\begin{array}{c}\text { Canadiens } \\
\%\end{array}$ \\
\hline Aucune & 28 & 28 \\
\hline 1 semaine de vacances & 23 & 9 \\
\hline 2 semaines de vacances & 30 & 30 \\
\hline 3 semaines de vacances & 8 & 23 \\
\hline 4 semaines de vacances & 5 & 4 \\
\hline Plus de 4 semaines & 6 & 6 \\
\hline TOTAL & $\mathbf{1 0 0}$ & $\mathbf{1 0 0}$ \\
\hline
\end{tabular}

\begin{tabular}{|c|c|c|}
\hline \multicolumn{3}{|c|}{ 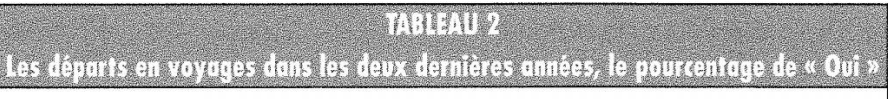 } \\
\hline Type de départ & $\begin{array}{l}\text { Américains } \\
\quad \%\end{array}$ & $\begin{array}{l}\text { Canadiens } \\
\quad \%\end{array}$ \\
\hline Une journée & 75 & 81 \\
\hline Quatre jours et plus & 80 & 71 \\
\hline
\end{tabular}

\begin{tabular}{|c|c|c|}
\hline \multicolumn{3}{|c|}{ 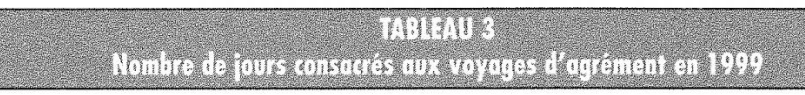 } \\
\hline Nombre de jours & $\begin{array}{c}\text { Américains } \\
\%\end{array}$ & $\begin{array}{c}\text { Canadiens } \\
\%\end{array}$ \\
\hline Aucun & 26 & 26 \\
\hline De 1 à 7 jours & 35 & 29 \\
\hline De 8 à 14 jours & 21 & 25 \\
\hline De 15 à 30 jours & 13 & 14 \\
\hline Plus de 30 jours & 5 & 6 \\
\hline TOTAL & 100 & 100 \\
\hline
\end{tabular}

\begin{tabular}{|c|c|c|}
\hline \multicolumn{3}{|c|}{ 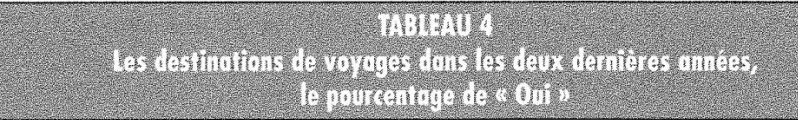 } \\
\hline Destination & $\begin{array}{c}\text { Américains } \\
\%\end{array}$ & $\begin{array}{c}\text { Canadiens } \\
\%\end{array}$ \\
\hline États-Unis & 76 & 31 \\
\hline Canada & 12 & 72 \\
\hline Autres pays & 26 & 18 \\
\hline
\end{tabular}

\begin{tabular}{|c|c|c|c|}
\hline \multicolumn{4}{|c|}{ 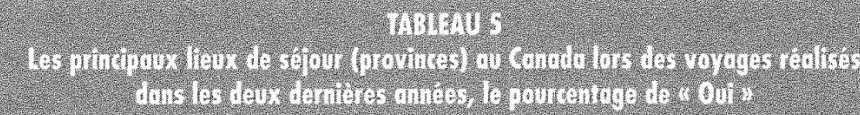 } \\
\hline Rang & Province & $\begin{array}{c}\text { Américains } \\
\%\end{array}$ & $\begin{array}{c}\text { Canadiens } \\
\%\end{array}$ \\
\hline 1 & Ontario & 44 & 28 \\
\hline 2 & Colombie-Britannique & 25 & 14 \\
\hline 3 & Québec & 17 & 14 \\
\hline
\end{tabular}


cerne les voyages des jeunes canadiens aux États-Unis, $89 \%$ y vont pour l'agrément ou la rencontre de parents et d'amis, $11 \%$ pour affaires et $8 \%$ pour des raisons personnelles. Les différences dans les motifs de voyages sont donc minimes dans les échanges touristiques entre les deux pays.

Au tableau 8 nous présentons les principales sources d'information pour la réalisation des voyages dans les deux dernières années. Nous remarquons que les jeunes canadiens lisent davantage les journaux pour obtenir de l'information sur les destinations de voyages ; la différence des pourcentages est d'environ $5 \%$ entre les jeunes des deux pays. En ce qui a trait à l'utilisation de l'Internet, les jeunes américains l'utilisent en plus grand nombre même si la proportion est très forte tant chez les Américains que les Canadiens (près de $60 \%$ ). Les jeunes américains ont utilisé plus souvent l'Internet pour faire des réservations (15\%) que les jeunes canadiens ( $5 \%$ ). L'Internet est donc une source d'information privilégiée des jeunes de ces deux pays.

\section{Une culture "jeunes" ou une culture commune?}

Dans l'ensemble, nous pouvons constater de profondes similitudes dans les comportements touristiques des jeunes des États-Unis et des jeunes du Canada : les jeunes voyagent beaucoup et utilisent en très grand nombre l'Internet comme principale source d'information sur les voyages.

Il y a tout de même certains points de divergence entre les jeunes des deux pays. Les principales variantes ressortent dans les éléments suivants :

- Les jeunes canadiens semblent profiter d'un nombre supérieur de semaines de vacances ;

- Les États-Unis exercent un attrait plus élevé sur les jeunes canadiens et, inversement, le Canada exerce un attrait plus faible (en comparaison) sur les jeunes américains ;

- Les jeunes américains voyagent plus en hiver et en été que pendant les autres saisons ;

- Les jeunes canadiens voyagent plus que les jeunes américains au printemps et à l'automne ;

- Les jeunes américains utilisent plus l'Internet pour faire des réservations de voyages que les jeunes canadiens.

Par ailleurs, en ce qui concerne les autres variables étudiées, les comportements touristiques des jeunes des États-Unis et des jeunes du Canada sont assez semblables. Au-delà des quelques éléments divergents, cités plus haut, il existe de grandes similitudes dans les comportements de voyages des jeunes de ces deux pays.

En conclusion, nous pouvons constater qu'il y a d'importantes affinités entre les jeunes des États-Unis et les jeunes du Canada, des ressemblances liées à une langue et à une culture communes («l'américanité »). Ces affinités renforcent les concordances

\begin{tabular}{|c|c|c|}
\hline \multicolumn{3}{|c|}{ 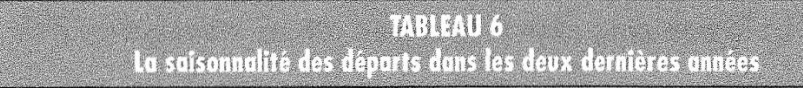 } \\
\hline Saison & $\begin{array}{l}\text { Américains } \\
\%\end{array}$ & $\begin{array}{c}\text { Canadiens } \\
\%\end{array}$ \\
\hline Hiver & 47 & 23 \\
\hline Printemps & 12 & 19 \\
\hline Été & 33 & 39 \\
\hline Automne & 8 & 19 \\
\hline TOTAL & 100 & 100 \\
\hline
\end{tabular}

\begin{tabular}{|c|c|c|}
\hline \multicolumn{3}{|c|}{ 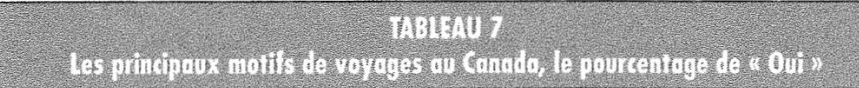 } \\
\hline Motifs de voyages au Canada & $\begin{array}{l}\text { Américains } \\
\%\end{array}$ & $\begin{array}{l}\text { Canadiens } \\
\%\end{array}$ \\
\hline $\begin{array}{l}\text { Pour l'agrément ou la rencontre } \\
\text { de parents et d'amis }\end{array}$ & 91 & 92 \\
\hline Pour affaires & 8 & 15 \\
\hline Pour des raisons personnelles & 12 & 17 \\
\hline
\end{tabular}

\begin{tabular}{|c|c|c|}
\hline \multicolumn{3}{|c|}{ 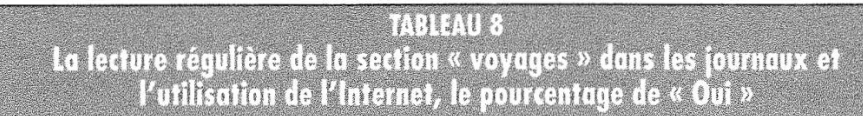 } \\
\hline $\begin{array}{l}\text { La lecture régulière de journaux } \\
\text { et l'utilisation de l'Internet }\end{array}$ & $\begin{array}{l}\text { Américains } \\
\%\end{array}$ & $\begin{array}{c}\text { Canadiens } \\
\%\end{array}$ \\
\hline Lecture quotidienne d'un journal & 19 & 24 \\
\hline $\begin{array}{l}\text { Lecture d'un journal en fin } \\
\text { de semaine }\end{array}$ & 21 & 27 \\
\hline $\begin{array}{l}\text { Utilisation régulière de l'Internet } \\
\text { comme source d'information }\end{array}$ & 68 & 58 \\
\hline $\begin{array}{l}\text { Réservation par le biais de } \\
\text { I'Internet pour un voyage au cours } \\
\text { des deux dernières années }\end{array}$ & 15 & 5 \\
\hline
\end{tabular}

et les rapprochements attribuables au groupe des dix-huit - vingtquatre ans. La « culture des jeunes » comme « habitus » propre à ce groupe d'âge, transcende-t-elle ces affinités particulières entre les jeunes des deux pays? Seule une étude comparative de plusieurs pays pourrait faire la lumière sur cette question.

Jean Stafford est professeur au Département d'études urbaines et touristiques de l'UQAM.

Bruno Sarrasin est chargé de cours au Département d'études urbaines et touristiques de l'UQAM. 